\title{
Single-fed Patch Antenna with Reconfigurable Orbit Angular Momentum Order
}

\author{
Liangcai ZHANG, Jianbo JIANG, Yongcong LIU, Weiwen LI \\ Dept. of Electronic Engineering, Xiamen University, 422 Siming South Road, Xiamen, China
}

13194086664@163.com, \{1693451556,1282227236\}@qq.com,wwl@xmu.edu.cn

Submitted May 22, 2020 / Accepted November 19, 2020

\begin{abstract}
Order reconfiguration of orbital angular momentum (OAM) is the foundation for wireless communications based on OAM state multiplexing. As the symmetry of a circular patch is disturbed by an arc segment, two degenerate modes can be synthesized under the single-fed condition to generate OAM waves. Due to arc segment independent of the radiation patch, its effective length is controlled by a switching diode to select different order degenerate modes for synthesis. Based on this idea, a reconfiguration of first-order and second-order OAM modes is achieved. In comparison, the performance of low-order OAM mode is better than that of high-order mode.
\end{abstract}

\section{Keywords}

Orbital angular momentum (OAM), reconfigurable antenna, patch antenna, degenerate mode

\section{Introduction}

Reconfigurable antennas achieve different functions through structural regulation, such as frequency reconfiguration, directional pattern reconfiguration and polarization reconfiguration, which have broad applications in wireless communication scenarios [1-3]. The use of reconfigurable vortex antenna to achieve multiplexing of orbital angular momentum (OAM) states can improve the spectrum utilization [4], [5]. However, it is controversial that OAM multiplexing is only a subset of MIMO (Multi-Input and MultiOutput) technologies, and cannot be effectively used to improve communication capacity in far-field links, although it has indisputable superiority in near-field applications [6]. In fact, it is also possible to realize customized structured fields or antenna radiation patterns of the topological robustness through the superposition of different order OAM modes [7]. In microwave and millimeter wave imaging, the resolution can be greatly improved by using OAM mode beams [8], [9].

According to the characteristics of electromagnetic vortex, there are mainly two types of reconfigurable OAM antennas, namely order and direction reconfiguration. Of course, these two types can also be used simultaneously
[10-12]. Reconfigurable antennas based on rotational states implement the OAM modes in two opposite direction at a fixed frequency. It is easy to realize the direction reconfiguration by using symmetrical antennas, but generally only three states can be formed, that is, left tright-handed rotation and zero-order OAM modes [10]. In theory, there are infinite OAM states in the same direction, but in reality it is difficult to obtain higher-order modes. Especially for OAM order reconfiguration at the same frequency, a combination of multiple size antennas may be required [13].

From the structural point of view, the antenna array is the easiest to realize the OAM mode reconfiguration, including the direction and order, but a complex feed network is required [14], [15]. A coding reflective metasurface can be used to generate the reconfigurable OAM mode through the programmable electronic control of each unit, but this control circuit is more complicated than the feed network [12]. For a single antenna of simple structure, OAM reconfiguration is usually performed at different frequencies [16]. In this paper, a circular patch with reconfigurable OAM order is constructed by controlling the length of a coupling arc segment to work at two frequencies.

\section{Antenna Structure}

Using spiral phase plates to modulate plane waves is a passive method for generating OAM modes, which is not easy to implement OAM order control [17], [18]. In fact, the synthesis of higher-order degenerate characteristic modes can directly radiate OAM modes [16], [19], [20]. For example, by superimposing degenerate $\mathrm{TM}_{21}$ or $\mathrm{TM}_{31}$ modes with an additional 90-degree phase, 1st or 2nd order OAM modes are obtained. A rotational symmetry patch can produce a 90-degree coupling phase between two degenerate TM modes through structural disturbance, and the OAM mode can be realized in the case of single feed. Thus, a single-fed circular patch antenna with an arc segment as a disturbance unit can radiate the OAM mode wave. Since the arc segment is independent of the radiation patch, it is easy to adjust the OAM order by controlling the length of the arc segment. Based on this, a switching diode is connected in series in the arc segment to electrically control the length of the arc segment, and the single-fed 


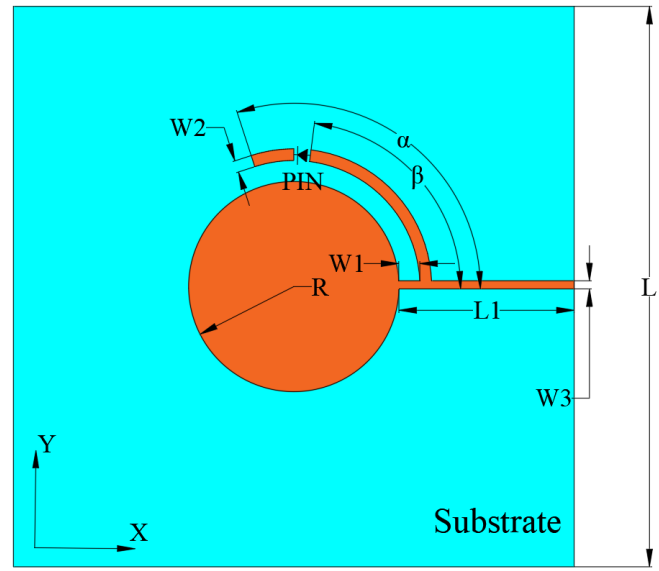

Fig. 1. Front view of reconfigurable antenna.

antenna with reconfigurable OAM order is constructed. The specific antenna structure is shown in Fig. 1.

The antenna is fabricated on a $48 \mathrm{~mm} \times 48 \mathrm{~mm}$ rectangular FR4 microwave substrate with a thickness of $1.5 \mathrm{~mm}$, a permittivity of 2.2 , and a loss tangent of 0.01 . The circular radiation patch with a radius $R=9 \mathrm{~mm}$ is etched in the middle of the substrate, which is fed by a horizontal microstrip line with a length $\mathrm{L} 1=15 \mathrm{~mm}$ and a width $\mathrm{W} 3=0.7 \mathrm{~mm}$. Outside the circular patch, there is a long arc ring starting from the microstrip feedline with a arc angle of $\alpha=0.6 \pi$, a distance from the circular patch of $\mathrm{W} 1=1.5 \mathrm{~mm}$, and a width of $\mathrm{W} 2=1 \mathrm{~mm}$. That is, the inner and outer radii of the arc ring are $10.5 \mathrm{~mm}$ and $11.5 \mathrm{~mm}$, respectively. In order to mount a switching diode, a cut with a width of $1.4 \mathrm{~mm}$ is introduced in the arc ring, and the arc angle between the cut and microstrip line is $\beta=0.46 \pi$. This arc section is called a short arc segment while the original arc is the long arc segment. In this regard, the length of the long arc segment is $21.7 \mathrm{~mm}$, and that of the short arc is $16.6 \mathrm{~mm}$.

Specifically, the diode BAR64-02V with the size of $1.2 \mathrm{~mm} \times 0.8 \mathrm{~mm} \times 0.55 \mathrm{~mm}$ is mounted at the cut of the arc segment, which occupies a small area and thus has little

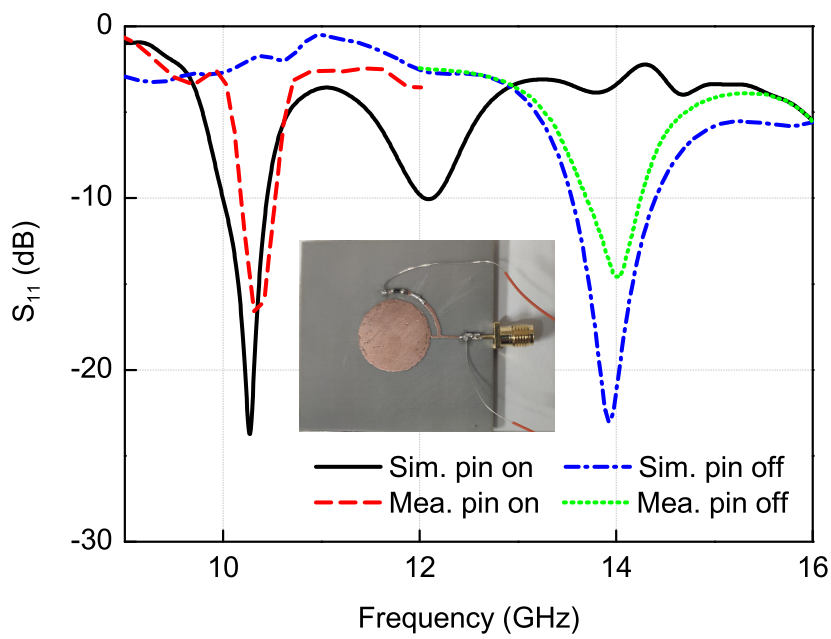

Fig. 2. Simulated and measured $S_{11}$ parameters of the proposed antenna. effect on the effective length of the arc segment. As no DC biasing voltage is applied, this diode exhibits high resistance characteristics. In the forward biased state the diode behaves almost as a short circuit and the long arc works. In the reverse biased state the diode behaves almost as an open circuit and only the short arc works. This diode has a high breakdown voltage and a low operating voltage, so it is easy to be controlled by a biasing circuit.

An antenna prototype is shown as the inset of Fig. 2. As the arc ring is switched on and off using PIN diode, the antenna can work in two OAM states, that is, the first-order mode at lower frequencies and the second-order mode at higher frequencies, to form reconfigurable OAM modes.

\section{1st Order OAM Mode}

As a forward biasing voltage is applied, the PIN diode is turned on and the long arc ring is effective. Thus, the antenna works at low frequencies. The simulated and tested $S_{11}$ results are shown in Fig. 2. The simulated frequency range of $S_{11}$ less than $-10 \mathrm{~dB}$ is $10.0-10.5 \mathrm{GHz}$, and the measured value is $10.2-10.6 \mathrm{GHz}$. The measured result is basically consistent with the simulated. The slight difference between them is because the simulation is performed under ideal conditions without considering the diode effects.

In this case, the phase and amplitude distributions of the electric field components $E_{\mathrm{x}}$ and $E_{\mathrm{y}}$ on the circumference with a radius of $20 \mathrm{~mm}$ in the plane with height of $15 \mathrm{~mm}$ from the antenna surface at $10.3 \mathrm{GHz}$ is shown in Fig. 3. It can be seen that the near-field electric field components in the $x$ and $y$ directions behave as first-order vortex modes, indicating that the antenna generates the first order OAM wave. Meanwhile, the phase and amplitude changes are relatively stable.

With the diode in the forward biased state, the radiation patterns in two orthogonal cut planes ( $x o z$ and yoz

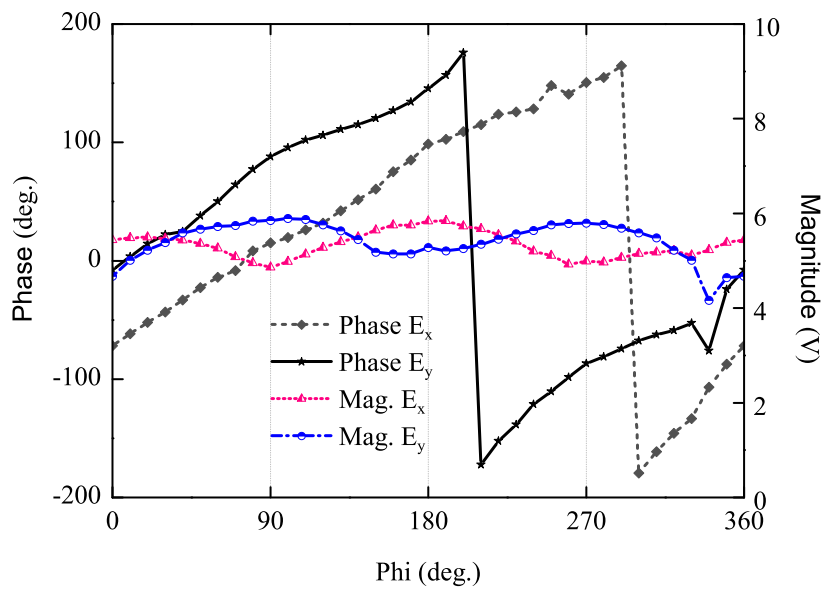

Fig. 3. The phase and amplitude distributions of the electric field components $E_{\mathrm{x}}$ and $E_{\mathrm{y}}$ on a circumference in a plane $15 \mathrm{~mm}$ away from the antenna surface at $10.3 \mathrm{GHz}$, as the diode is in the forward biased state. 


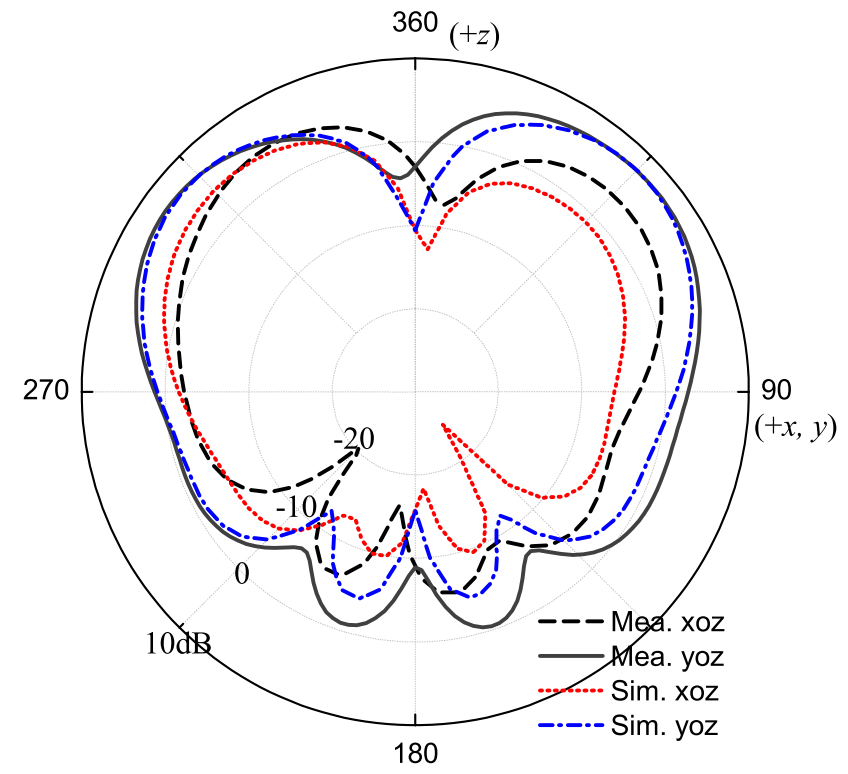

Fig. 4. Antenna patterns at $10.3 \mathrm{GHz}$ with the diode in the forward biased state.

planes) at $10.3 \mathrm{GHz}$ are shown in Fig. 4. An obvious minimum value of the field strength appears near the beam propagation $z$ axis, which is the characteristic of the firstorder OAM wave. In fact, the dents appearing near the $z$ axis correspond to the phase singularities. Certainly, since the feedline and arc segment affect the structural symmetry, the antenna pattern symmetry is slightly broken. However, due to the influence of the diode and its DC control wires, the minimum field intense points at boresight in the measured patterns deviate further from the $+z$ axis than in the simulated.

\section{2nd Order OAM Mode}

As a reverse biasing voltage is applied, the PIN diode is turned off and the short arc ring mainly affects the radiation field characteristics. It can be seen from Fig. 1 that the antenna works at higher frequencies in that case. The simulated frequency range of $S_{11}$ less than $-10 \mathrm{~dB}$ is $13.5-14.4 \mathrm{GHz}$, and the measured value is $13.7-14.3 \mathrm{GHz}$. Also due to the diode influence, there is a certain difference between the simulated and measured values. At this time, the original first-order OAM mode at lower frequencies cannot be effectively radiated. In fact, while the first-order mode works, the second-order mode at higher frequencies cannot be generated. It is in line with the requirements of reconfigurable antennas.

In that case, the phase and amplitude distributions of the electric field components $E_{\mathrm{x}}$ and $E_{\mathrm{y}}$ on the circumference with a radius of $20 \mathrm{~mm}$ in the plane with a height of $15 \mathrm{~mm}$ from the antenna surface at $14.0 \mathrm{GHz}$ is shown in Fig. 5. It can be clearly seen that they exhibit a $4 \pi$ phase shift, indicating that the 2nd OAM wave is generated. At the same time, the amplitude fluctuations are relatively large, indicating that the purity of the second-order OAM

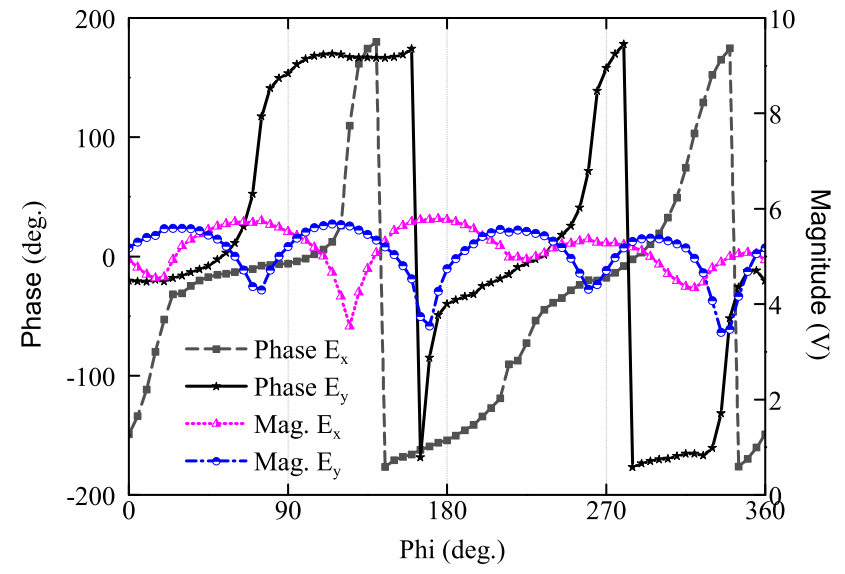

Fig. 5. The phase and amplitude distributions of the electric field components $E_{\mathrm{x}}$ and $E_{\mathrm{y}}$ on a circumference in a plane $15 \mathrm{~mm}$ away from the antenna surface at 14.0 $\mathrm{GHz}$, as the diode is in the reverse biased state.

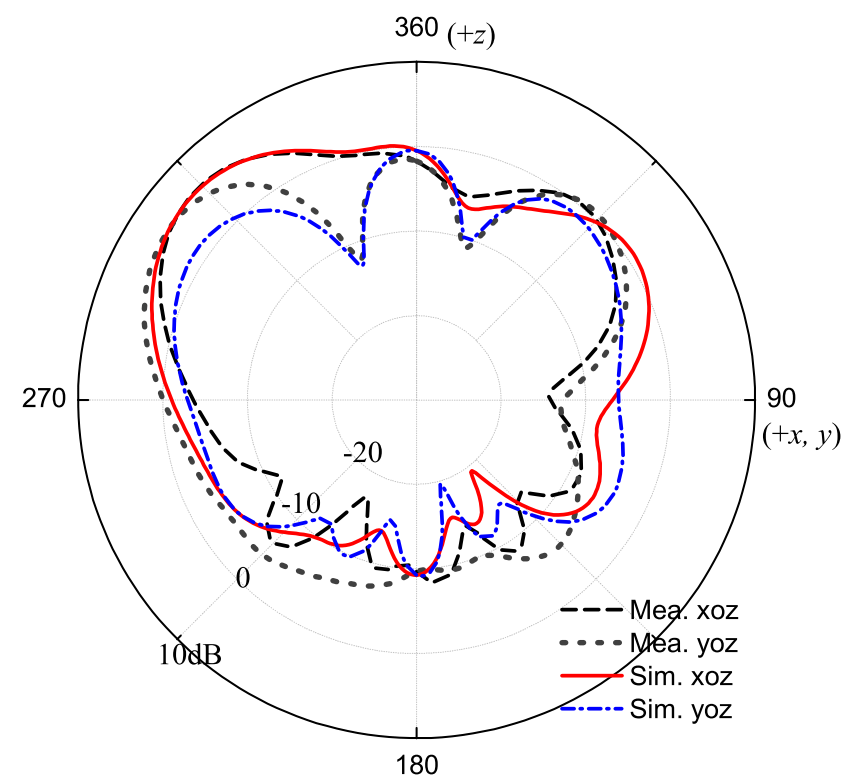

Fig. 6. Radiation patterns at $14.0 \mathrm{GHz}$ with the diode in the reverse biased state.

mode is lower than that of the first-order. From the perspective of mode synthesis, it is more difficult to generate high-order OAM waves due to the increase in parasitic modes, and their performance will be degraded.

With the diode in the reverse biased state, the radiation patterns in two orthogonal cut planes at $14.0 \mathrm{GHz}$ is shown in Fig. 6. It can be seen that the maximum radiation direction does not appear in the $+z$ axis direction. However, the minimum field strength is also not shown in the $+z$ direction, which occurs at the theta angle of about 20 degree due to the effect of antenna symmetry. In that case, the main lobe is more deviated from the minimum position, which is exactly the characteristic of second-order OAM mode. Similarly, due to the influence of the DC bias voltage and its connecting wires, the measured patterns are a bit different from the simulated, but the overall trend is consistent. 


\section{Analysis and Discussion}

The proposed antenna can radiate first-order OAM mode at $10.3 \mathrm{GHz}$ and second-order OAM wave at 14.0 GHz through controlling the biasing voltage of the diode. To further clarify the generation mechanism of the OAM modes from the perspective of the radiation source, the full-wave electric field distributions on the antenna surface at two bands are shown in Fig. 7. It can be seen that the $\mathrm{TM}_{21}$ mode is formed at $10.3 \mathrm{GHz}$, and the $\mathrm{TM}_{31}$ mode is shown at $14.0 \mathrm{GHz}$. Therefore, it is confirmed that the first-order OAM mode is synthesized from the superposition of two degenerate $\mathrm{TM}_{21}$ modes, while the secondorder OAM mode is from two degenerate $\mathrm{TM}_{31}$ modes.

Considering the substrate influence, the wavelength at the low frequency point of $10.3 \mathrm{GHz}$ can correspond to the $21.7 \mathrm{~mm}$ length of the long arc. This indicates that as there is a strong electric field at the microstrip feed point of circular patch, the arc end also has a strong electric field distribution. Thus, the $\mathrm{TM}_{21}$ mode can be effectively excited, as shown in Fig. 7(a). Correspondingly, as shown in Fig. 7(b), the short arc can effectively excite the $\mathrm{TM}_{31}$ mode.

Comparing two OAM modes, the phase variations with the azimuth angle at the low frequency are more uniform than that at the high frequency. At higher frequencies,
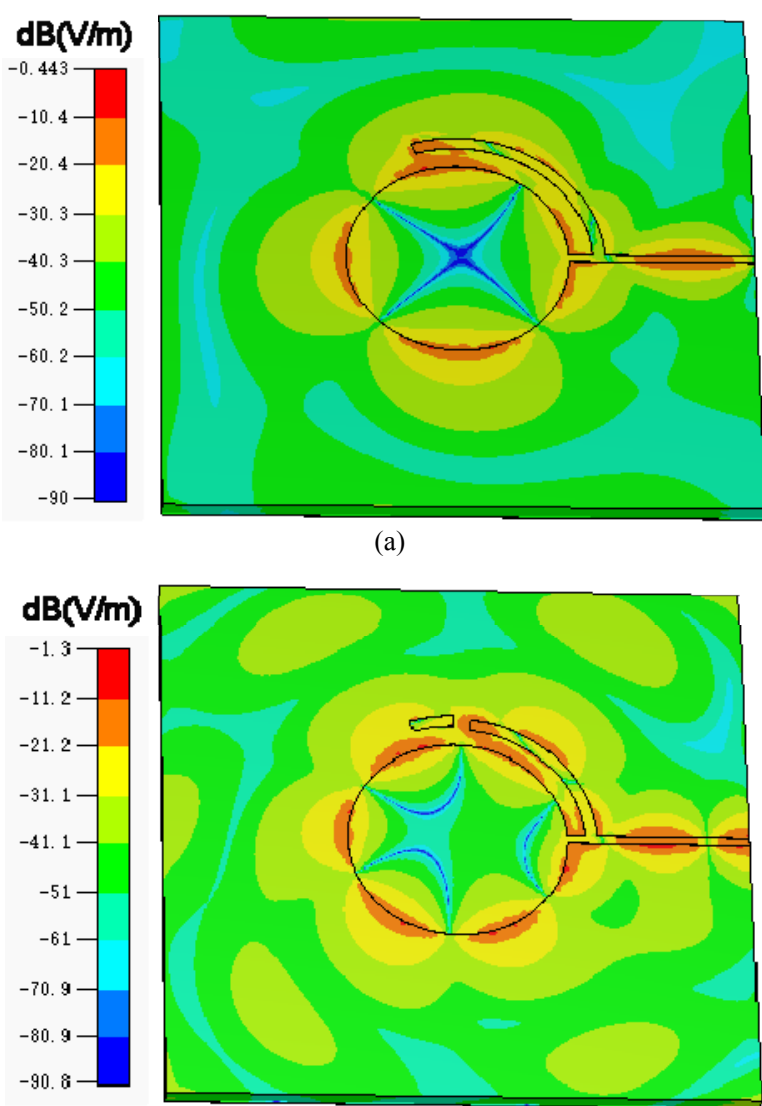

(b)

Fig. 7. Full wave electric field distributions on the antenna surface for (a) 1 st order OAM at $10.3 \mathrm{GHz}$ and (b) 2nd order OAM at $14.0 \mathrm{GHz}$.

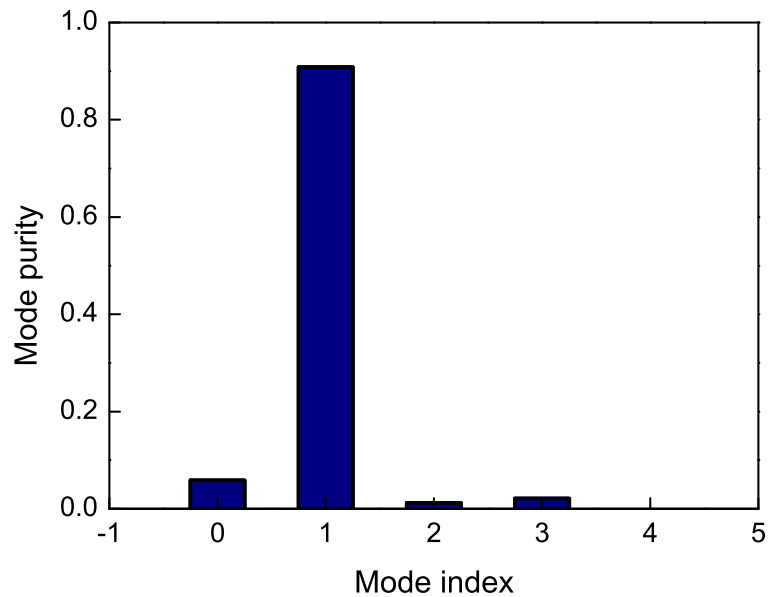

(a)

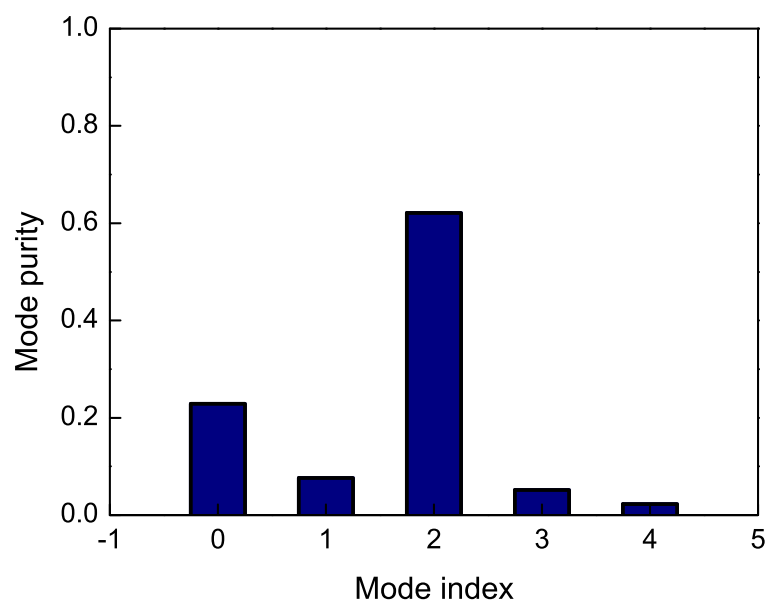

(b)

Fig. 8. Mode purity for (a) 1st order OAM at $10.3 \mathrm{GHz}$ and (b) 2nd order OAM at $14.0 \mathrm{GHz}$.

the unconnected arc conductor does not act as a disturbance unit, but it can produce a reflection effect on the radiation field. Meanwhile, it influences the electric field distribution at the end of the short arc section, which further destroys the field symmetry.

In order to quantify the performance comparison of these two OAM modes, Fourier transform method [21] is used to analyze the mode purity of OAM waves. According to the phase and amplitude distributions of the field components in Figs. 3 and 5, the mode purity of two OAM states as shown in Fig. 8 is obtained. It can be seen that the mode purity of the first-order OAM wave reaches $90 \%$, while that for the second-order mode is only about $65 \%$. Generally, for a single antenna structure, the mode purity of the high-order OAM wave is low, which is detrimental to the effective application of OAM modes.

\section{Conclusion}

Using a circular patch with a controllable arc segment, a reconfigurable single-fed antenna with two OAM states is constructed. If zero order is also considered, threestate reconfiguration can be achieved. Since this antenna 
has the rotation symmetry, if the arc segments are loaded on both sides of the feedline, 5-state reconfiguration can be applied. This antenna structure is simple and easy to implement, and thus can be conveniently applied to the wireless communication systems based on OAM mode multiplexing.

\section{Acknowledgments}

This work was supported by the Natural Science Foundation of China (NSFC) under Grant No. 62071403, by the Natural Science Foundation of Fujian Province of China under Grant No. 2019J01045, and by the Open Fund of the State Key Laboratory of Integrated Optoelectronics, Jilin University, under Grant No. IOSKL2017KF02. The authors would also like to acknowledge the anonymous reviewers for their helpful comments.

\section{References}

[1] SHIRAZI, M., HUANG, J., LI, T., et al. A switchable-frequency slot-ring antenna element for designing a reconfigurable array. IEEE Antennas and Wireless Propagation Letters, 2018, vol. 17, no. 2, p. 229-233. DOI: 10.1109/LAWP.2017.2781463

[2] LIN, W., WONG, H., ZIOLKOWSKI, R. W. Wideband patternreconfigurable antenna with switchable broadside and conical beams. IEEE Antennas and Wireless Propagation Letters, 2017, vol. 16, p. 2863-2641. DOI: 10.1109/LAWP.2017.2738101

[3] ZHU, H. L., CHEUNG, S. W., LIU, X. H., et al. Design of polarization reconfigurable antenna using metasurface. IEEE Transactions on Antennas and Propagation, 2014, vol. 62, no. 6, p. 2891-2898. DOI: 10.1109/TAP.2014.2310209

[4] TAMBURINI, F., MARI, E., SPONSELli, A., et al. Encoding many channels on the same frequency through radio vorticity: first experimental test. New Journal of Physics, 2012, vol. 14, p. 1-17. DOI: $10.1088 / 1367-2630 / 14 / 3 / 033001$

[5] TAMBURINI, F., MARI, E., PARISI, G., et al. Tripling the capacity of a point-to-point radio link by using electromagnetic vortices. Radio Science, 2015, vol. 50, no. 6, p. 501-508. DOI: $10.1002 / 2015 \mathrm{RS} 005662$

[6] MORABITO, A. F., DI DONATO, L., ISERNIA, T. Orbital angular momentum antennas: Understanding actual possibilities through the aperture antennas theory. IEEE Antennas and Propagation Magazine, 2018, vol. 60, no. 2, p. 59-67. DOI: 10.1109/MAP.2018.2796445

[7] BARBUTO, M., MIRI, M., ALU, A., et al. A topological design tool for the synthesis of antenna radiation patterns. IEEE Transactions on Antennas and Propagation, 2020, vol. 68, no. 3, p. 1851-1859. DOI: 10.1109/TAP.2019.2944533

[8] WANG, J., LIU, K., CHENG, Y., et al. Vortex SAR imaging method based on OAM beams design. IEEE Sensors Journal, 2019 vol. 19, no. 24, p. 11873-11879. DOI: 10.1109/JSEN.2019.2937976

[9] LIU, K., LI, X., GAO, Y., et al. Microwave imaging of spinning object using orbital angular momentum. Journal of Applied Physics, 2017, vol. 122, p. 1-6. DOI: 10.1063/1.4991655

[10] SHI, H., WANG, L., PENG, G., et al. Generation of multiple modes microwave vortex beams using active metasurface. IEEE
Antennas and Wireless Propagation Letters, 2019, vol. 18, no. 1, p. 59-63. DOI: 10.1109/LAWP.2018.2880732

[11] KANG, L., LI, H., ZHOU, J., et al. A mode-reconfigurable orbital angular momentum antenna with simplified feeding scheme. IEEE Transactions on Antennas and Propagation, 2019, vol. 67, no. 7, p. 4866-4871. DOI: 10.1109/TAP.2019.2916595

[12] HAN, J., LI, L., YI, H., et al. 1-bit digital orbital angular momentum vortex beam generator based on a coding reflective metasurface. Optical Materials Express, 2018, vol. 8, no. 11, p. 3470-3478. DOI: 10.1364/OME.8.003470

[13] ZHANG, Z., XIAO, S., LI, Y., et al. A circularly polarized multimode patch antenna for the generation of multiple orbital angular momentum modes. IEEE Antennas and Wireless Propagation Letters, 2017, vol. 16, p. 521-524. DOI: 10.1109/LAWP.2016.2586975

[14] DENG, C., ZHANG, K., FENG, Z. Generating and measuring tunable orbital angular momentum radio beams with digital control method. IEEE Transactions on Antennas and Propagation, 2017, vol. 65, no. 2, p. 899-902. DOI: 10.1109/TAP.2016.2632532

[15] MOHAMMADI, S. M., DALDORFF, L. K. S., BERGMAN, J. E. $\mathrm{S}$., et al. Orbital angular momentum in radio - a system study. IEEE Transactions on Antennas and Propagation, 2010, vol. 58, no. 2 , p. 565-572. DOI: 10.1109/TAP.2009.2037701

[16] LI, W., ZHANG, L., ZHU, J., et al. Constructing dual-frequency OAM circular patch antenna using characteristic mode theory. Journal of Applied Physics, 2019, vol. 126, p. 064501-1-6. DOI: 10.1063/1.5100631

[17] YU, S., LI, L., SHI, G. Dual-polarization and dual-mode orbital angular momentum radio vortex beam generated by using reflective metasurface. Applied Physics Express, 2016, vol. 9, no. 8 , p. 082202-1-4. DOI: 10.7567/APEX.9.082202

[18] KOU, N., YU, S., LI, L. Generation of high-order Bessel vortex beam carrying orbital angular momentum using multilayer amplitude-phase-modulated surfaces in radiofrequency domain. Applied Physics Express, 2017, vol. 10, no. 1, p. 016701-1-4. DOI: 10.7567/APEX.10.016701

[19] CABEDO-FABRES, M., ANTONINO-DAVIU, E., VALERONOGUEIRA, A., et al. The theory of characteristic modes revisited: A contribution to the design of antennas for modern applications. IEEE Antennas and Propagation Magazine, 2007, vol. 49, no. 5, p. 52-68. DOI: 10.1109/map.2007.4395295

[20] BARBUTO, M., TROTTA, F., BILOTTI, F., et al. Circular polarized patch antenna generating orbital angular momentum. Progress In Electromagnetics Research, 2014, vol. 148, p. 23-30. DOI: $10.2528 /$ pier14050204

[21] YAO, E., FRANKE-ARNOLD, S., COURTIAL, J., et al. Fourier relationship between angular position and optical orbital angular momentum. Optics Express, 2006, vol. 14, no. 20, p. 9071-9076. DOI: $10.1364 / \mathrm{OE} .14 .009071$

\section{About the Authors ...}

Liangcai ZHANG was born in China in 1995. He received his B.S. degree from Jimei University (JMU), Xiamen, Fujian, China in 2018. At present he is pursuing the M.S. degree in Electronics and Communication Engineering. His research interests are antenna applications and microwave circuits.

Jianbo JIANG was born in China in 2000. He is currently pursuing the B.E. degree at Xiamen University, China. His 
current research interests include integrated circuit design and electromagnetic fields.

Yongcong LIU was born in China in 1995. He received his B.S. degree from Xidian University (XDU), Xi'an, Shaanxi, China in 2017. At present he is pursuing his M.S. degree in Electronics and Communication Engineering. His research interests are antenna technology and antenna design.
Weiwen LI (corresponding author) received the B.S. degree in Electronic Engineering from Jilin University, Changchun, China, in 1993, the M.S. degree in Material Engineering and the Ph.D. degree in Electronic Engineering from Zhejiang University, Hangzhou, China, in 2002 and 2005, respectively. He is currently an Associate Professor of Electronic Engineering, Xiamen University, China. His research interest includes the antenna theory and design. 